\title{
Decreasing the Adverse Effects of Endotracheal Suctioning During Mechanical Ventilation by Changing Practice
}

\author{
Salvatore Maurizio Maggiore MD PhD, François Lellouche MD, Claudia Pignataro MD, \\ Emmanuelle Girou PharmD, Bernard Maitre MD, Jean-Christophe M Richard MD PhD, \\ François Lemaire MD, Christian Brun-Buisson MD, and Laurent Brochard MD
}

\begin{abstract}
BACKGROUND: Little is known about the incidence of and risk factors for adverse effects from endotracheal suctioning. We studied the incidence and risk factors, and evaluated the effect of suctioning practice guidelines. METHODS: During a 3-month period, in 79 mechanically ventilated subjects, we recorded the adverse effects in 4,506 suctioning procedures. Then practice guidelines were implemented, and 1 year later, during another 3-month period, in 68 subjects, we recorded the adverse effects in 4,994 suctioning procedures. RESULTS: In the first period, adverse effects occurred frequently: oxygen desaturation in $46.8 \%$ of subjects and $6.5 \%$ of suctionings, hemorrhagic secretions in $31.6 \%$ of subjects and $4 \%$ of suctionings, blood pressure change in $24.1 \%$ of subjects and $1.6 \%$ of suctionings, and heart rate change in $10.1 \%$ of subjects and $1.1 \%$ of suctionings. After guidelines implementation, all complications, both separately and all together, were reduced. The incidence of all complications together decreased from $59.5 \%$ to $42.6 \%$ of subjects, and from $12.4 \%$ to $4.9 \%$ of procedures (both $P<.05$ ). $\mathrm{PEEP}>5 \mathrm{~cm} \mathrm{H}_{2} \mathrm{O}$ was an independent risk factor for oxygen desaturation. Receiving $>\mathbf{6}$ suctionings per day was a risk factor for desaturation and hemorrhagic secretions. The use of guidelines was independently associated with fewer complications. CONCLUSIONS: Endotracheal suctioning frequently induces adverse effects. Technique, suctioning frequency, and higher PEEP are risk factors for complications. Their incidence can be reduced by the implementation of suctioning guidelines. Key words: endotracheal suctioning; closed suctioning system; practice guidelines; mechanical ventilation; ARDS; PEEP. [Respir Care 2013; 58(10):1588-1597. (C) 2013 Daedalus Enterprises]
\end{abstract}

\section{Introduction}

The presence of an artificial airway during mechanical ventilation makes coughing less effective or not possible.

\footnotetext{
Dr Maggiore is affiliated with the Department of Anesthesiology and Intensive Care, Agostino Gemelli Hospital, Catholic University of the Sacred Heart, Rome, Italy. Drs Lellouche, Pignataro, Richard, Lemaire, Brun-Buisson, and Brochard are affiliated with the Medical ICU; Dr Girou is affiliated with the Infection Control Unit; and Dr Maitre is affiliated with the Department of Pulmonology, Henri Mondor University Hospital, Créteil, France. Dr Lellouche is also affiliated with the Cardiac Surgery ICU, Laval University Hospital, Québec City, Canada. Dr Pignataro is also affiliated with the Department of Anesthesiology and Intensive Care, Lariboisière Hospital, Paris, France. Drs Richard and Brochard are also affiliated with the Intensive Care Department, University Hospital, University of Geneva, Geneva, Switzerland. Dr Brochard is also affiliated with the Institut National de la Santé et de la Recherche Médicale (INSERM) Unit 955, Paris 12 University, Créteil, France.
}

Endotracheal suctioning is therefore needed to avoid accumulation of secretions into the lung, and its associated complications. Nevertheless, endotracheal suctioning is an invasive procedure, and is not free from hazards and, exceptionally, from lethal adverse events. ${ }^{1}$ Numerous side

\footnotetext{
Dr Maggiore presented a version of this paper at the International Conference of the American Thoracic Society, held May 17-22, 2002, in Atlanta, Georgia.

The authors have disclosed no conflicts of interest.

Correspondence: Salvatore Maurizio Maggiore MD PhD, Department of Anesthesiology and Intensive Care, Agostino Gemelli Hospital, Catholic University of the Sacred Heart, Largo Agostino Gemelli 8, 00168 Rome, Italy. E-mail: smmaggiore@ rm.unicatt.it.
}

DOI: $10.4187 /$ respcare. 02265 


\section{Decreasing the Adverse EfFects of Endotracheal Suctioning}

effects of endotracheal suctioning have been reported.2-11 Some old studies on selected patient populations suggested a high frequency of specific adverse events, such as oxygen desaturation and arrhythmia.1,12 Leur and coworkers ${ }^{13}$ reported a relatively low incidence of some endotracheal suctioning adverse events in a selected population of surgical patients without ARDS and with a short duration of mechanical ventilation. Thus, the incidence and risk factors of adverse effects of endotracheal suctioning in a general medical population of critically ill patients are uncertain.

\section{See the Related Editorial on Page 1707}

In 2010 the American Association for Respiratory Care published updated clinical practice guidelines for endotracheal suctioning, ${ }^{9}$ with the aim of optimizing the procedure and reducing the hazards. Specific suctioning strategies are not systematically used in ICUs, ${ }^{14}$ and their usefulness has not been well assessed. Moreover, the optimal approach to reduce endotracheal-suctioning-related complications has not been fully clarified. ${ }^{15,16}$ Therefore we carried out a clinical investigation to evaluate the incidence of endotracheal-suctioning-associated adverse events in mechanically ventilated patients, and to determine whether the implementation of practice guidelines could decrease the rate. Suctioning-induced adverse events before and after the implementation of practice guidelines were compared using the same methodology. Practice guidelines for endotracheal suctioning were drafted independently from those of the American Association for Respiratory Care, ${ }^{9}$ and before their release.

\section{Methods}

\section{Study Location and Subject Population}

The study was conducted in the 26-bed medical ICU of Henri Mondor University Hospital, Créteil, France. The institutional ethics committee approved the study and waived the requirement for informed consent. All consecutive patients needing mechanical ventilation and $\geq 18$ years old were included during two 3-month periods, from February to April 2000 (period 1) and from April to June 2001 (period 2). According to clinical requirements, subjects received sedation by continuous infusion, following our local protocol, which was the same in the 2 study periods. They were mechanically ventilated with volume controlled continuous mandatory ventilation or pressure support mode. Heat and moisture exchangers were generally used. In all subjects, pulse oximetry, electrocardiography, and arterial blood pressure were continuously monitored, according to routine practice.

\section{QUICK LOOK}

\section{Current knowledge}

Endotracheal suctioning is associated with various complications, ranging from discomfort to hemodynamic collapse, but the incidence and severity of these complications has not been systematically studied. The usefulness of adherence to suctioning guidelines has also not been addressed.

\section{What this paper contributes to our knowledge}

Adverse effects of suctioning, particularly oxygen desaturation and hemorrhagic secretions, were frequent and were reduced by the implementation of practice guidelines. Factors that increased the risk of suctioningrelated complications included more frequent suctioning, requirement for a PEEP of $>5 \mathrm{~cm} \mathrm{H}_{2} \mathrm{O}$, and the presence of ARDS.

\section{Study Design and Data Collection}

Endotracheal-suctioning-related adverse events were collected daily during the 23 -month periods. During the first period, endotracheal suctioning was performed according to the usual practice at that time: suctioning procedures were mainly performed routinely every 2 hours or more often if secretions were visible in the endotracheal/ tracheostomy tube; subjects were disconnected from the ventilator; the duration of the procedure, the vacuum pressure (frequently $>400 \mathrm{~cm} \mathrm{H}_{2} \mathrm{O}$ ), size of the suction catheter, and depth of suctioning were not standardized; saline was instilled in case of dry, tenacious secretions; no special precaution was used in subjects with ARDS; in general, closed suction systems were not used.

In the 1-year interval between the 2 study periods, clinical practice guidelines for endotracheal suctioning were developed during the first month based on the available evidence, and subsequently implemented. The rationale and the feasibility of each guideline were discussed in depth with physicians and nurses until a consensus was reached and, finally, guidelines were described in a written protocol. To facilitate implementation, repeated meetings were organized to educate and to instruct the whole personnel about this protocol. Repeated informal follow-up training was also performed, and a medical referent was always available for any questions and technical needs.

In the second period, endotracheal suctioning was performed according to practice guidelines. No major change took place in the unit in between these 2 periods regarding airway and ventilator management. During the 2 study periods, nurses were instructed to detect and report daily 


\section{Decreasing the Adverse EfFects of Endotracheal Suctioning}

on standardized data collection sheets all adverse events for each suctioning procedure. During and just after the intervention period (period 2), adherence to practice guidelines was also assessed by respiratory therapists and physicians not involved in suctioning procedures. They randomly observed suctioning procedures and, for each procedure, reported if guidelines were followed. A total of 600 observations were performed during night and day. Nurses were not informed of the observers' task.

Adherence to the study protocol was assessed daily by investigators and respiratory therapists. This was done by comparing the number of suctioning procedures reported on the subject's daily clinical chart and the number of procedures reported on the specific daily sheet used for the study. In addition, in an attempt to validate the reliability of detecting and reporting adverse effects of endotracheal suctioning following the given instructions, one of the investigators repeatedly observed suctioning procedures and reported if these instructions were followed. Reliability in reporting adverse events of suctioning was calculated as:

Number of correctly reported events/number of observations $\times 100$

A total of 540 observations were performed: 270 in each period.

\section{Clinical Practice Guidelines for Endotracheal Suctioning}

The guidelines for endotracheal suctioning were as follows:

- Frequency of Endotracheal Suctioning. The suctioning procedures had to be performed according to the subject's needs, and not routinely. ${ }^{17}$ The need for endotracheal suctioning was evaluated based on oscillations on the expiratory part of the flow-time curve ${ }^{18}$ and tracheal or bronchial respiratory sounds. ${ }^{19,20}$ Ventilator alarms (increased peak airway pressure during volume controlled continuous mandatory ventilation, or decreased tidal volume during pressure-targeted ventilation modes), presence of secretions in the endotracheal tube or oxygen desaturation, after excluding other possible causes, were also considered as later indicators of the need for suctioning. In paralyzed subjects, endotracheal suctioning was performed every 4 hours, even if the aforementioned signs were absent.

- Disconnection from the ventilator had to be avoided. The suction catheter was introduced through the swivel adapter of the catheter mount, or a closed system was used. ${ }^{6}$
- Depth of Endotracheal Suctioning. To minimize mucosal trauma, shallow suction (limited to the artificial airway and the trachea) was performed, instead of deep suctioning. ${ }^{13}$ In practice, approximately $8-10 \mathrm{~cm}$ of the suction catheter was left outside the endotracheal tube. With a tracheostomy the suction catheter was introduced up to approximately half its length. In any case, insertion was stopped if an obstacle was met, and the suction catheter was withdrawn approximately $1 \mathrm{~cm}$. Suctioning was then started while gradually withdrawing the catheter.

- Instillation of saline was avoided. ${ }^{21-23}$ In case of dry, tenacious secretions, the heat and moisture exchanger was replaced by a heated humidifier. Selective suctioning under direct visualization by fiberoptic bronchoscopy was performed if a mucus plug was suspected.

- Size of the Suction Catheter. This had to be adapted to the size of the endotracheal tube, so that the diameter of the suction catheter was $<50 \%$ the inner diameter of the artificial airway. ${ }^{10,11,17,24,25}$ In practice, 16 French suction catheters were used with artificial airways with an inner diameter $\geq 9 \mathrm{~mm}$, 14 French suction catheters were used with $8.0-\mathrm{mm}$ or $8.5-\mathrm{mm}$ endotracheal tubes, and 12 French catheters with $7.0-\mathrm{mm}$ or $7.5-\mathrm{mm}$ endotracheal tubes.

- The duration of endotracheal suctioning was limited to $<10-15$ seconds. ${ }^{17,26,27}$ If needed, the suctioning procedure was repeated after a time period sufficient for restoring baseline ventilation and oxygen saturation.

- The suction pressure had to be set between 200 and $250 \mathrm{~mm} \mathrm{Hg} .17,25,27,28$

- In subjects with ARDS, to minimize suctioning-induced lung derecruitment, a closed suction system was used, and ventilator auto-triggering was allowed during the procedure. ${ }^{5,6}$ Closed suctioning systems were changed in case of mechanical failure or visible soiling only: not routinely. ${ }^{23,29-31}$ Recruitment maneuvers were used in case of persisting hypoxemia after suctioning.,432

A sterile technique was employed at all times. The subject's appearance (eg, sweating, skin color, agitation), vital signs (oxygen saturation, heart rate, cardiac rhythm, arterial blood pressure), and ventilatory parameters (breathing frequency, tidal volume, peak inspiratory pressure) were monitored during the whole suctioning procedure. ${ }^{17,27}$

\section{Adverse Effects of Endotracheal Suctioning}

Adverse effects of endotracheal suctioning were defined a priori, as follows: 
- Oxygen desaturation: an $\mathrm{S}_{\mathrm{pO}_{2}}$ decrease of $>5 \%$

- Hemorrhagic secretions: blood visible in suctioned secretions

- Severe hypertension: an increase in systolic blood pressure to $>200 \mathrm{~mm} \mathrm{Hg}$

- Severe hypotension: a drop in systolic blood pressure to $<80 \mathrm{~mm} \mathrm{Hg}$

- Severe tachycardia: an increase in heart rate to $>150$ beats/min

- Severe bradycardia: a decrease in heart rate to $<50$ beats/ $\min$

- Arrhythmia: any new appearance of sustained supraventricular or ventricular arrhythmia

\section{Statistical Analysis}

Results are reported as mean $\pm \mathrm{SD}$, except when otherwise indicated. Incidence density, expressed per 100 ventilator days, was calculated according to the formula:

(Number of events/study days) $\times 100$

Dichotomous variables were compared with use of the chi-square test, and continuous variables with the Student $t$ test. After assessing normality, the continuous variables were dichotomized using adequate cut-points. Logistic regression analysis was performed, incorporating all factors with $P<.10$ in the univariate analysis. A $P \leq .05$ in a 2-tailed test was used to indicate significance. All analyses were performed using statistics software (StatView 5, SAS Institute, Cary, North Carolina).

\section{Results}

We included 147 subjects, and 9,500 suctioning procedures were recorded during a total of 1,225 ventilator days. During the pre-intervention 3-month period (period 1), 4,506 suctioning procedures in 79 subjects were collected during 604 ventilator days. After guidelines implementation (period 2), 4,994 suctioning procedures, in 68 subjects, were collected during 621 ventilator days.

Nurse reliability in detecting and reporting suctioning adverse effects was $94 \%$ overall, varying from $91 \%$ in period 1 to $96 \%$ in period 2 . The most common errors in reporting adverse events concerned severe hypotension $(20 \%)$ and oxygen desaturation (17\%). The comparison between the number of suctioning procedures reported on the subject's daily chart and the number of procedures reported on the specific daily sheet used for the study showed that endotracheal suctioning procedures were ad-
Table 1. Subjects, Outcomes, and Number of Suctioning Procedures

\begin{tabular}{|c|c|c|c|}
\hline & $\begin{array}{c}\text { Before } \\
\text { Guidelines } \\
n=79\end{array}$ & $\begin{array}{c}\text { After } \\
\text { Guidelines } \\
n=68\end{array}$ & $P$ \\
\hline Age, y & $57.7 \pm 17$ & $60.2 \pm 15.5$ & .36 \\
\hline SAPS II at admission & $46.1 \pm 16.5$ & $50.5 \pm 20.3$ & .16 \\
\hline \multicolumn{4}{|l|}{ Admission type, $\%$} \\
\hline Medical & 74.7 & 79.4 & \\
\hline Surgical & 8.9 & 5.9 & .74 \\
\hline Emergency surgery & 16.5 & 14.7 & \\
\hline \multicolumn{4}{|l|}{ Diagnoses, $\%$} \\
\hline Respiratory failure & 29.1 & 29.4 & .97 \\
\hline Sepsis or septic shock & 27.8 & 17.6 & .14 \\
\hline ARDS & 13.9 & 23.5 & .13 \\
\hline Heart failure & 15.2 & 5.9 & .07 \\
\hline Cardiac arrest & 5.1 & 8.8 & .37 \\
\hline Hemorrhagic shock & 1.3 & 7.4 & .06 \\
\hline Cerebrovascular disease & 2.5 & 5.9 & .31 \\
\hline Pulmonary embolism & 1.3 & 1.5 & .91 \\
\hline Neurological disease & 3.8 & 0 & .10 \\
\hline Duration of mechanical ventilation, $\mathrm{d}$ & $10.9 \pm 12.2$ & $14.5 \pm 19.5$ & .18 \\
\hline ICU stay, d & $17.3 \pm 15.4$ & $23.3 \pm 32.2$ & .14 \\
\hline ICU survival, \% & 65 & 49 & .05 \\
\hline Suctioning procedures, no. & 4,506 & 4,994 & .23 \\
\hline Suctioning procedures/subject/d & $6.6 \pm 2.2$ & $6.7 \pm 2.8$ & .91 \\
\hline Subjects with $>6$ suctionings $/ \mathrm{d}, \%$ & 65.8 & 51.5 & .08 \\
\hline Ventilator days, no. & 604 & 621 & .34 \\
\hline Ventilator days/subject & $7.7 \pm 7.8$ & $9.1 \pm 10.8$ & .34 \\
\hline
\end{tabular}

\pm values are mean $\pm \mathrm{SD}$

SAPS $=$ Simplified Acute Physiology Score

equately reported in $95.2 \%$ of cases, varying from $94.8 \%$ in period 1 to $95.6 \%$ in period 2 . As shown in Table 1, the general characteristics of the subjects, number of collected suctioning procedures, and outcomes were not statistically different between the 2 periods.

\section{Adverse Effects of Endotracheal Suctioning Before Guidelines Implementation}

In period 1, 47 subjects $(59.5 \%)$ experienced at least one complication from endotracheal suctioning: oxygen desaturation occurred in 37 subjects, hemorrhagic secretions in 25 , hypertension in 14 , hypotension in 7 , tachycardia in 5, and bradycardia in 4 (Fig. 1). One subject experienced transient ventricular tachycardia, which resolved spontaneously after suctioning. Adverse effects occurred in 559 procedures (Fig. 2) and 173 ventilator days (Fig. 3), which calculated to a rate of endotrachealsuctioning-associated adverse effects of 92.5 per 100 ventilator days. Oxygen desaturation (incidence density of 48.3 per 100 ventilator days) and hemorrhagic secretions (incidence density of 30 per 100 ventilator days) were the most frequent adverse effects. 


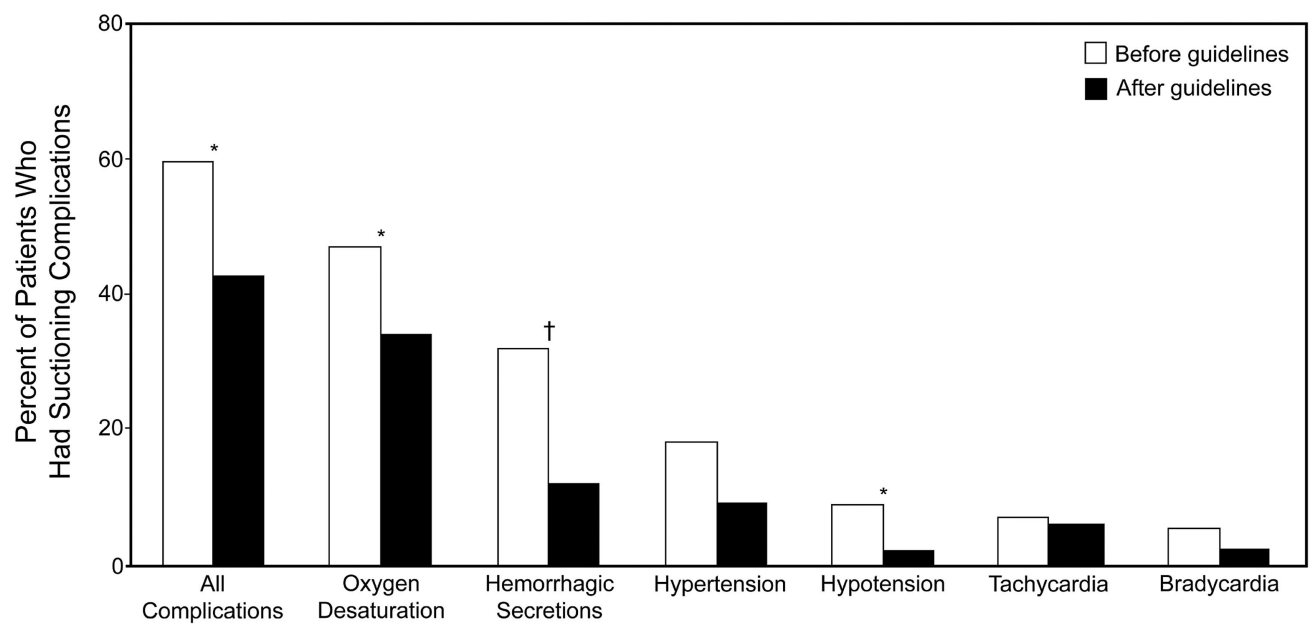

Fig. 1. Mean percentages of subjects who suffered adverse effects from endotracheal suctioning. The sum of proportions for specific complications is greater than the percentage for all complications, because several complications could occur with a single procedure. ${ }^{*} P<.05 . \dagger P=004$.

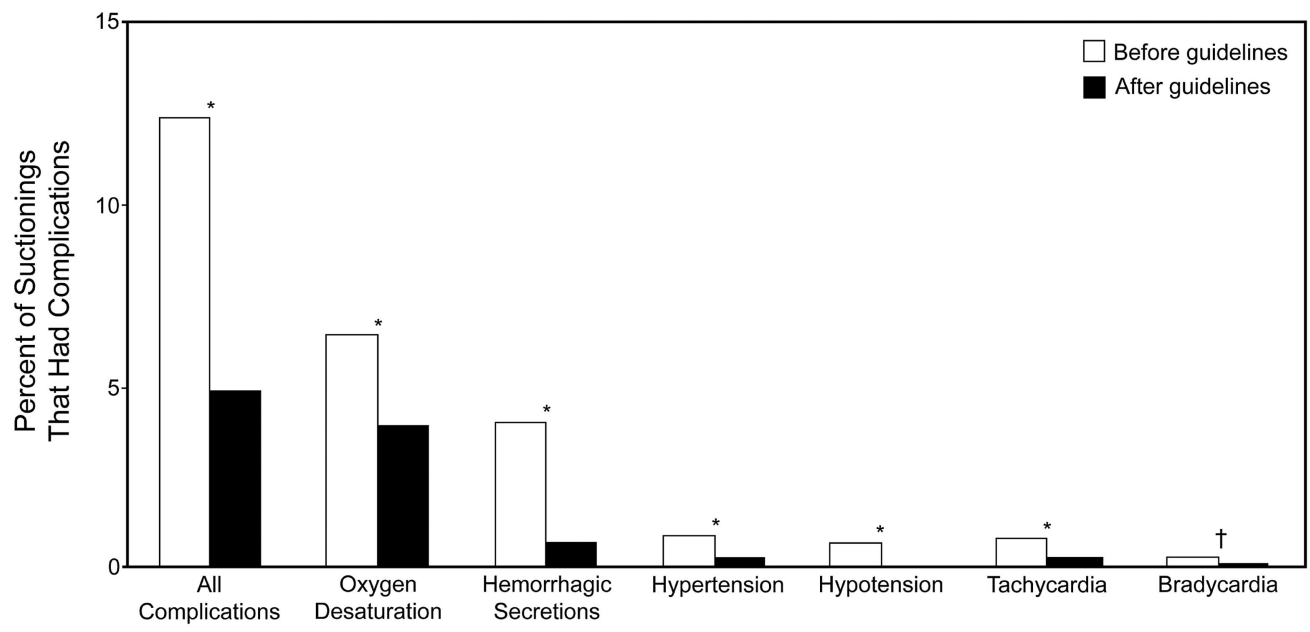

Fig. 2. Mean percentages of suctioning procedures that had complications. The sum of proportions for specific complications is greater than the percentage for all complications, because several complications could occur during a single procedure. ${ }^{*} P<.001$. $† P=.006$

\section{Effect of Practice Guidelines on Endotracheal- Suctioning-Associated Adverse Effects}

Adherence to practice guidelines was $95.9 \%$. The effects of guidelines implementation are shown in Figures 1, 2 , and 3. Compared to period 1 , the proportion of subjects experiencing any complication from endotracheal suctioning was significantly reduced after guidelines implementation $(P=.04)$. Particularly, fewer subjects presented hemorrhagic secretions $(P=.004)$, hypotension $(P=.04)$, and, after adjusting for the duration of mechanical ventilation, oxygen desaturation $(P=.02)$. No subject presented any form of arrhythmia during period 2 . The rate of complicated suctioning procedures was reduced by $61 \%$ in period 2, with a rate of endotracheal-suctioning-associated adverse effects of 39 per 100 ventilator days $(P<.001)$. This reduction concerned all adverse effects, with a decrease of $40 \%$ for oxygen desaturation (incidence density of 31.1 per 100 ventilator days $)(P<.001), 83 \%$ for hemorrhagic secretions (5.5 per 100 ventilator days) $(P<$ $.001), 78 \%$ for hypertension (1.8 per 100 ventilator days) $(P<.001), 94 \%$ for hypotension $(0.3$ per 100 ventilator days) $(P<.001), 75 \%$ for tachycardia $(1.8$ per 100 ventilator days $)(P<.001)$, and $67 \%$ for bradycardia $(0.6$ per 100 ventilator days $)(P=.006)$. The proportion of days of mechanical ventilation with complicated suctioning procedures was also significantly reduced in period 2 $(P<.001)$. Oxygen desaturation and hemorrhagic secretions remained the most frequent adverse effects. In period 2 the proportion of subjects with frequent suction- 


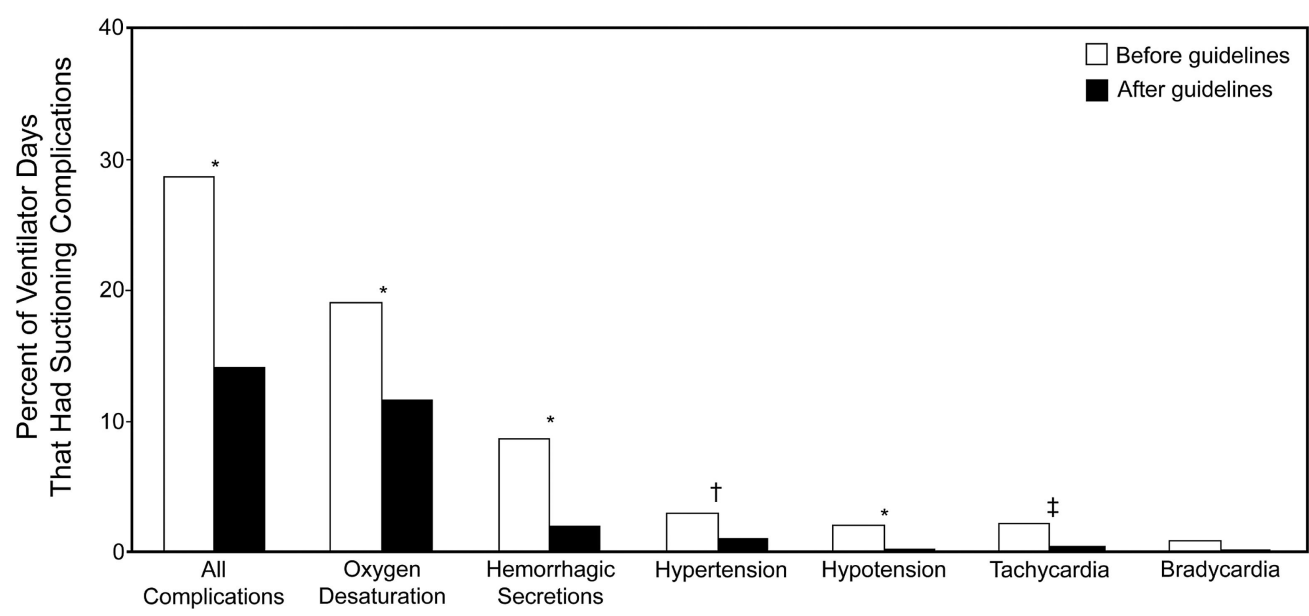

Fig. 3. Mean percentages of ventilator days on which suctioning procedures had complications. The sum of proportions for specific complications is greater than the percentage for all complications, because several complications could occur during a single procedure. ${ }^{*} P<.001$. $\dagger P=.01 \ddagger P=.05$.

ing procedures $(>6 / \mathrm{d})$ was lower, albeit not significantly, than in period 1 (see Table 1).

\section{Risk Factors for Endotracheal-Suctioning-Associated Adverse Effects}

For the analysis, hypertension and hypotension were grouped together into a "blood pressure changes" category, and tachycardia and bradycardia were grouped into a "heart rate changes" category. The results of the univariate analysis for individual and grouped adverse effects are shown in Table 2. General characteristics were not different between subjects with or without endotrachealsuctioning-associated adverse effects. For all adverse events, frequency of suctioning was significantly higher in subjects with adverse effects than those without. Subjects exhibiting oxygen desaturation during endotracheal suctioning had a higher frequency of ARDS, were ventilated with a PEEP higher than $5 \mathrm{~cm} \mathrm{H}_{2} \mathrm{O}$, and had an $\mathrm{F}_{\mathrm{IO}_{2}}$ greater than 0.6 more frequently than subjects who did not present oxygen desaturation. Anticoagulation for at least 3 days was more frequent in subjects with hemorrhagic secretions than in those who did not present such complication.

The results of the multivariate regression analysis are shown in Table 3. Only PEEP $>5 \mathrm{~cm} \mathrm{H}_{2} \mathrm{O}$ and $>6 / \mathrm{d}$ suctioning procedures were independently associated with an increased risk of oxygen desaturation during suctioning. By contrast, not having ARDS and being in period 2 were independent protective factors for desaturation. Frequency of suctioning $(>6 / \mathrm{d})$, but not anticoagulation, was independently associated with an increased risk of hemorrhagic secretions, while period 2 was a protective factor for this complication. The only independent risk factor for suctioning-induced blood pressure changes was the occurrence of oxygen desaturation.

\section{Discussion}

The main results of this study are that:

- Endotracheal suctioning was frequently complicated, mainly by oxygen desaturation and hemorrhagic secretions.

- The implementation of practice guidelines reduced the incidence of all adverse effects.

- Frequent suctioning, PEEP $>5 \mathrm{~cm} \mathrm{H}_{2} \mathrm{O}$, and ARDS were risk factors for the main adverse events (oxygen desaturation and hemorrhagic secretions).

- Oxygen desaturation was a risk factor for hemodynamic alterations during endotracheal suctioning.

The first step of our education initiative to reduce adverse effects from endotracheal suctioning was to assess their incidence during current practice. In this study a large proportion of mechanically ventilated subjects experienced adverse events when endotracheal suctioning was not protocolized. Previous small clinical studies have reported several complications of endotracheal suctioning. ${ }^{1,3,5,6,12}$ In a population of mostly surgical patients with a relatively short duration of mechanical ventilation $(4-5 \mathrm{~d})$ and without ARDS, Leur et al found that complications of routine endotracheal suctioning occurred in $38.6 \%$ of procedures. ${ }^{13}$ When a less invasive suctioning technique was adopted (using a modified suction catheter), complications were reduced to $28.6 \%$. In the first period of our study (routine suctioning), we found a smaller incidence of hypertension and arrhythmia, but a larger incidence of oxygen desaturation than previously reported. ${ }^{13}$ Differences in the defi- 
Table 2. Univariate Analysis of Complications of Endotracheal Suctioning

\begin{tabular}{|c|c|c|c|}
\hline & $\begin{array}{c}\text { Subjects } \\
\text { With Adverse } \\
\text { Events }\end{array}$ & $\begin{array}{c}\text { Subjects } \\
\text { Without Adverse } \\
\text { Events }\end{array}$ & $P$ \\
\hline Oxygen desaturation & $(n=60)$ & $(n=87)$ & \\
\hline Age, mean \pm SD & $59.6 \pm 16.5$ & $58.3 \pm 16.3$ & .65 \\
\hline SAPS II, mean \pm SD & $46.3 \pm 16.1$ & $49.4 \pm 19.9$ & .32 \\
\hline ICU survival, \% & 58.3 & 56.3 & .81 \\
\hline ARDS, $\%$ & 25 & 13.8 & .08 \\
\hline PEEP $>5 \mathrm{~cm} \mathrm{H}_{2} \mathrm{O}, \%$ & 58.3 & 33.3 & .003 \\
\hline $\mathrm{F}_{\mathrm{IO}_{2}}>0.6, \%$ & 66.7 & 41.4 & .003 \\
\hline$>6$ suctionings/d, $\%$ & 81.7 & 43.7 & $<.001$ \\
\hline Period $1 / 2^{*}$ & $37 / 23$ & $42 / 45$ & .02 \\
\hline Hemorrhagic secretions & $(n=33)$ & $(n=114)$ & \\
\hline Age, mean $\pm \mathrm{SD}$ & $56.3 \pm 16.7$ & $59.6 \pm 16.2$ & .32 \\
\hline SAPS II, mean \pm SD & $44.3 \pm 13.9$ & $49.3 \pm 19.4$ & .17 \\
\hline ICU survival, $\%$ & 60.6 & 56.1 & .65 \\
\hline Anticoagulation $>3$ days, $\%$ & 36.4 & 21.9 & .09 \\
\hline$>6$ suctionings $/ \mathrm{d}, \%$ & 84.9 & 51.8 & $<.001$ \\
\hline Period $1 / 2$ & $25 / 8$ & $54 / 60$ & .004 \\
\hline Blood pressure changes $\dagger$ & $(n=26)$ & $(n=121)$ & \\
\hline Age, mean $\pm \mathrm{SD}$ & $56.1 \pm 16.4$ & $59.4 \pm 16.3$ & .35 \\
\hline SAPS II, mean \pm SD & $45 \pm 16.1$ & $48.8 \pm 18.9$ & .35 \\
\hline ICU survival, \% & 73.1 & 53.7 & .07 \\
\hline Oxygen desaturation, $\%$ & 73.1 & 33.9 & $<.001$ \\
\hline$>6$ suctionings $/ \mathrm{d}, \%$ & 80.8 & 54.5 & .01 \\
\hline Period $1 / 2$ & $19 / 7$ & $60 / 61$ & .03 \\
\hline Heart rate changes $\ddagger$ & $(n=13)$ & $(n=134)$ & \\
\hline Age, mean \pm SD & $60.6 \pm 16.6$ & $58.7 \pm 16.4$ & .69 \\
\hline SAPS II, mean \pm SD & $49.1 \pm 19.5$ & $48.1 \pm 18.4$ & .85 \\
\hline ICU survival, $\%$ & 46.2 & 58.2 & .40 \\
\hline Oxygen desaturation, $\%$ & 76.9 & 37.3 & .006 \\
\hline Hemodynamic changes, $\%$ & 38.5 & 15.7 & .04 \\
\hline$>6$ suctionings $/ \mathrm{d}, \%$ & 92.3 & 56 & .01 \\
\hline Period $1 / 2$ & $8 / 5$ & $71 / 63$ & .55 \\
\hline \multicolumn{4}{|c|}{$\begin{array}{l}\text { * After adjustment for the duration of mechanical ventilation. } \\
\uparrow \text { The blood pressure changes category includes hypertension and hypotension. } \\
\text { † The heart rate changes category includes tachycardia and bradycardia. } \\
\text { SAPS II = Simplified Acute Physiology Score II } \\
\text { Period } 1=\text { before implementation of guidelines } \\
\text { Period } 2 \text { = after implementation of guidelines }\end{array}$} \\
\hline
\end{tabular}

nition of complications, in suctioning techniques, and in patient population likely explain these discrepancies. Instead of relative changes, we used absolute cutoff values for blood pressure and heart rate modifications, to facilitate the task of reporting adverse effects for nurses. As a consequence, we could have underestimated these complications. Not surprisingly, we found a greater incidence of oxygen desaturation than in Leur's study, in which patients with severe acute respiratory failure were excluded. ${ }^{13}$ Our subjects were sicker, and approximately $20 \%$ of them had ARDS (see Table 1). In particular, their illness severity was slightly greater, although not significantly, in period 2, as suggested by the slightly higher Simplified
Acute Physiology Score II and greater incidence of ARDS. This can probably explain the somewhat longer duration of mechanical ventilation and ICU stay, and the trend toward lower ICU survival in period 2 (see Table 1).

Our data suggest the usefulness of practice guidelines to reduce the hazards of endotracheal suctioning. ${ }^{9,11,23}$ In particular, our results support the clinical value of the recently updated clinical practice guidelines of the American Association for Respiratory Care. ${ }^{9}$ Our guidelines, independently developed on the basis of available evidence, are in fact very similar, although there may be some differences, mainly related to the control of the depth of suctioning. Our method consisted of leaving approximately $8-10 \mathrm{~cm}$ 
Table 3. Multivariate Logistic Regression Analysis

\begin{tabular}{llcc}
\hline \hline & $\begin{array}{c}\text { Odds } \\
\text { Ratio }\end{array}$ & $95 \% \mathrm{CI}$ & $P$ \\
\hline Oxygen desaturation $(n=60)$ & & & \\
$\quad \mathrm{PEEP}>5 \mathrm{~cm} \mathrm{H}_{2} \mathrm{O}$ & 2.96 & $1.26-6.95$ & .01 \\
$>6$ suctionings/d & 6 & $2.54-14.23$ & $<.001$ \\
$\mathrm{~F}_{\mathrm{IO}_{2}}>0.6$ & 2.25 & $0.99-5.07$ & .052 \\
$\quad \mathrm{No}$ ARDS & 0.31 & $0.1-0.9$ & .03 \\
$\quad$ Period 2 & 0.4 & $0.17-0.93$ & .03 \\
Hemorrhagic secretions $(n=33)$ & & & \\
$\quad$ Anticoagulation $>3$ days & 1.45 & $0.58-3.64$ & .43 \\
$>6$ suctionings/d & 4.25 & $1.45-12.44$ & .008 \\
$\quad$ Period 2 & 0.31 & $0.13-0.78$ & .01 \\
Blood pressure changes $(n=26) *$ & & & \\
$\quad$ Oxygen desaturation & 4 & $1.46-11$ & .007 \\
$\quad>6$ suctionings/d & 1.88 & $0.6-5.86$ & .28 \\
Period 2 & 0.44 & $0.16-1.17$ & .09 \\
& & & \\
* The blood pressure changes category includes hypertension and hypotension. & \\
Period 2 = After implementation of guidelines & & & \\
\hline
\end{tabular}

of the suction catheter outside the endotracheal tube, or, in the extreme case of a too deep insertion of the suction catheter inside the trachea so that an obstacle was met, of withdrawing the suction catheter before applying the negative pressure. This method may be imprecise for determining suction depth, and it does not precisely reflect the recent clinical practice guidelines of the American Association for Respiratory Care. ${ }^{9}$ The use of suction catheters with length marks would be the best solution to perform shallow suctioning. Unfortunately, we did not have these catheters available in our ICU, as is still the case in many ICUs. Our protocol, including the technique of suctioning, was designed to make the individual tasks as easy as possible with the available means. Nevertheless, we observed a quite striking decrease in the rate of hemorrhagic secretions in period 2, suggesting that a lower rate of mucosal trauma should have occurred after the implementation of guidelines, and supporting the idea that the depth of suctioning was indeed reduced in period 2. The bleeding rate could have been even lower with a more precise control of the depth of suctioning. Our study design did not permit us to determine the weight of each recommendation on the global impact of guidelines implementation on adverse effects of endotracheal suctioning.

Endotracheal-suctioning-induced oxygen desaturation results from lung derecruitment secondary to both the loss of positive airway pressure due to ventilator disconnection and the application of negative pressure, particularly in patients with ARDS. $3,5,6,11$ The duration of the suctioning procedure, the level of the applied negative pressure, the size of the suction catheter, and instillation of saline may also influence the occurrence of lung derecruitment and hypoxia. ${ }^{10,11,25-27}$ Accordingly, the partial prevention of derecruitment obtained by avoiding ventilator disconnection or using a closed system in ARDS patients, 5,6,11 while limiting the duration of procedure, the level of the negative suctioning pressure, and the size of the suction catheter, can explain the observed decrease in oxygen saturation after guidelines implementation. The presence of blood in suctioned secretions is likely explained by airway mucosal trauma caused by repeated introductions of the suction catheter and application of negative pressure. In agreement with a previous study, ${ }^{13}$ the reduced depth of suctioning and the limitation of negative pressure provided by our protocol can account for the large decrease in the rate of hemorrhagic secretions in period 2 . A further limitation of the suction pressure might have been associated with a further reduction of oxygen desaturation and hemorrhagic secretions, but this might have also reduced the efficacy of suctioning in clearing secretions. Blood pressure and heart rate modifications can result from abrupt changes of intrathoracic pressure, the release of endogenous catecholamines secondary to suctioning-induced stress, hypoxemia, and vagal stimulation..$^{1,13,33}$

To our knowledge this is the first study assessing risk factors for adverse effects of endotracheal suctioning. This may be useful in identifying patients at increased risk for suctioning-related complications. We found that subjects with ARDS, and subjects ventilated with high PEEP, were at an increased risk of oxygen desaturation (see Table 3). We have previously shown in ARDS subjects that lung derecruitment observed after ventilator disconnection was correlated with the level of applied PEEP. ${ }^{6}$ Here we could quantify the degree of hypoxemic risk conferred by PEEP $>5 \mathrm{~cm} \mathrm{H}_{2} \mathrm{O}$ (a 196\% increase in risk). In addition, we showed that frequent suctioning procedures $(>6 / \mathrm{d})$ substantially increase the risk of oxygen desaturation and hemorrhagic secretions (see Table 3). No risk factor was found for heart rate changes, likely because of their low incidence, whereas oxygen desaturation was the only identified prognostic factor for arterial blood pressure alterations (see Table 3). This confirms previous data suggesting that hypoxemia plays a key role for the occurrence of this complication. ${ }^{33}$

The 2 suctioning procedures were applied sequentially, in 2 different periods, and not randomized. Although randomization might have allowed a more rigorous study design, the contemporaneous use of 2 different suctioning procedures would have been a source of confusion for the nursing staff, potentially leading to major protocol deviations. In addition, the study was designed as an education initiative, and the study protocol was designed to make the individual tasks as easy as possible. The high adherence obtained with this approach may have compensated 


\section{Decreasing the Adverse EfFects of Endotracheal Suctioning}

at least in part for the less rigorous study design. We did not compare directly the efficacy of the 2 suctioning procedures, which would require a different study design. All our recommendations were based on the literature, however, and on our own experiments. ${ }^{6}$

\section{Conclusions}

The adverse effects of endotracheal suctioning, particularly oxygen desaturation and hemorrhagic secretions, are frequent and can be reduced by the implementation of practice guidelines. Several factors can be used to identify patients at higher risk of airway-suctioning-related complications so that we can pay more attention to high-risk patients and target future intervention studies toward those patients most likely to benefit.

\section{ACKNOWLEDGMENTS}

We thank Graça Salgueiro and Véronique Morisset (nurses) and Sylvie Lely and Nicole Jackson (respiratory therapists) for their constant support, and Jérôme Pigeot and Solenne Taillé (biomedical engineers) for technical assistance.

\section{REFERENCES}

1. Shim C, Fine N, Fernandez R, Williams MH Jr. Cardiac arrhythmias resulting from tracheal suctioning. Ann Intern Med 1969;71(6): 1149-1153.

2. McCauley CS, Boller LR. Bradycardic responses to endotracheal suctioning. Crit Care Med 1988;16(11):1165-1166.

3. Brochard L, Mion G, Isabey D, Bertrand C, Messadi AA, Mancebo $\mathrm{J}$, et al. Constant-flow insufflation prevents arterial oxygen desaturation during endotracheal suctioning. Am Rev Respir Dis 1991; 144(2):395-400.

4. Lu Q, Capderou A, Cluzel P, Mourgeon E, Abdennour L, LawKoune JD, et al. A computed tomographic scan assessment of endotracheal suctioning-induced bronchoconstriction in ventilated sheep. Am J Respir Crit Care Med 2000;162(5):1898-1904.

5. Cereda M, Villa F, Colombo E, Greco G, Nacoti M, Pesenti A. Closed system endotracheal suctioning maintains lung volume during volume-controlled mechanical ventilation. Intensive Care Med 2001;27(4):648-654.

6. Maggiore SM, Lellouche F, Pigeot J, Taillé S, Deye N, Durrmeyer $\mathrm{X}$, et al. Prevention of endotracheal suctioning-induced alveolar derecruitment in acute lung injury. Am J Respir Crit Care Med 2003; 167(9):1215-1224.

7. Lindgren S, Almgren B, Högman M, Lethvall S, Houltz E, Lundin S, et al. Effectiveness and side effects of closed and open suctioning: an experimental evaluation. Intensive Care Med 2004;30(8):1630-1637.

8. Seymour CW, Cross BJ, Cooke CR, Gallop RL, Fuchs BD. Physiologic impact of closed-system endotracheal suctioning in spontaneously breathing patients receiving mechanical ventilation. Respir Care 2009;54(3):367-374.

9. American Association for Respiratory Care. Clinical Practice Guidelines. Endotracheal suctioning of mechanically ventilated patients with artificial airways 2010. Respir Care 2010;55(6):758-764.

10. Tingay DG, Copnell B, Grant CA, Dargaville PA, Dunster KR, Schibler A. The effect of endotracheal suction on regional tidal ventilation and end-expiratory lung volume. Intensive Care Med 2010; 36(5):888-896.
11. Maggiore SM, Volpe C. Endotracheal suctioning in hypoxemic patients. Réanimation 2011;20(1):12-18.

12. Adlkofer RM, Powaser MM. The effect of endotracheal suctioning on arterial blood gases in patients after cardiac surgery. Heart Lung 1978;7(6):1011-1014.

13. Leur JP, Zwaveling JH, Loef BG, Schans CP. Endotracheal suctioning versus minimally invasive airway suctioning in intubated patients: a prospective randomised controlled trial. Intensive Care Med 2003;29(3):426-432.

14. Sole ML, Byers JF, Ludy JE, Zhang Y, Banta CM, Brummel K. A multisite survey of suctioning techniques and airway management practices. Am J Crit Care 2003;12(3):220-230.

15. Subirana M, Sola I, Benito S. Closed tracheal suction systems versus open tracheal suction systems for mechanically ventilated adult patients. Cochrane Database Syst Rev 2007;(4):CD004581.

16. Grivans C, Lindgren S, Aneman A, Stenqvist O, Lundin S. A Scandinavian survey of drug administration through inhalation, suctioning and recruitment maneuvers in mechanically ventilated patients. Acta Anaesthesiol Scand 2009;53(6):710-716.

17. American Association for Respiratory Care. Clinical Practice Guideline. Endotracheal suctioning of mechanically ventilated adults and children with artificial airways. Respir Care 1993;38(5):500-504.

18. Jubran A, Tobin MJ. Use of flow-volume curves in detecting secretions in ventilator-dependent patients. Am J Respir Crit Care Med 1994;150(3):766-769.

19. Guglielminotti J, Alzieu M, Maury E, Guidet B, Offenstadt G. Bedside detection of retained tracheobronchial secretions in patients receiving mechanical ventilation: is it time for tracheal suctioning? Chest 2000;118(4):1095-1099.

20. Lucchini A, Zanella A, Bellani G, Gariboldi R, Foti G, Pesenti A, et al. Tracheal secretion management in the mechanically ventilated patient: comparison of standard assessment and an acoustic secretion detector. Respir Care 2011;56(5):596-603.

21. Ackerman MH. The effect of saline lavage prior to suctioning. Am J Crit Care 1993;2(4):326-330.

22. Hagler DA, Traver GA. Endotracheal saline and suction catheters: sources of lower airway contamination. Am J Crit Care 1994;3(6): 444-447.

23. Pedersen CM, Rosendahl-Nielsen M, Hjermind J, Egerod I. Endotracheal suctioning of the adult intubated patient-what is the evidence? Intensive Crit Care Nurs 2009;25(1):21-30.

24. Tiffin NH, Keim MR, Frewen TC. The effects of variations in flow through an insufflating catheter and endotracheal-tube and suctioncatheter size on test-lung pressures. Respir Care 1990;35(9):889-897.

25. Morrow BM, Futter MJ, Argent AC. Endotracheal suctioning: from principles to practice. Intensive Care Med 2004;30(6):1167-1174.

26. Rindfleisch SH, Tyler ML. Duration of suctioning: an important variable. Respir Care 1983;28(5):457-459.

27. Hess DR, Kacmarek RM. Technical aspects of the patient-ventilator interface. In: Tobin MJ, editor. Principles and practice of mechanical ventilation, 1st edition. New York: McGraw-Hill; 1994:1039-1065.

28. Lasocki S, Lu Q, Sartorius A, Fouillat D, Remerand F, Rouby JJ. Open and closed-circuit endotracheal suctioning in acute lung injury: efficiency and effects on gas exchange. Anesthesiology 2006;104(1): 39-47.

29. Kollef MH, Prentice D, Shapiro SD, Fraser VJ, Silver P, Trovillion $\mathrm{E}$, et al. Mechanical ventilation with or without daily changes of inline suction catheters. Am J Respir Crit Care Med 1997;156(2 Pt 1): 466-472.

30. Maggiore SM. Endotracheal suctioning, ventilator-associated pneumonia, and costs: open or closed issue? Intensive Care Med 2006; 32(4):485-487. 
31. Lorente L, Lecuona M, Jiménez A, Mora ML, Sierra A. Tracheal suction by closed system without daily change versus open system. Intensive Care Med 2006;32(4):538-544.

32. Heinze H, Eichler W, Karsten J, Sedemund-Adib B, Heringlake M, Meier T. Functional residual capacity-guided alveolar recruitment strategy after endotracheal suctioning in cardiac surgery patients. Crit Care Med 2011;39(5):1042-1049.

33. Winston SJ, Gravelyn TR, Sitrin RG. Prevention of bradycardic responses to endotracheal suctioning by prior administration of nebulized atropine. Crit Care Med 1987;15(11):1009-1011.

This article is approved for Continuing Respiratory Care Education credit. For information and to obtain your CRCE

(free to AARC members) visit

www.rcjournal.com 